Session 2793

\title{
Ethical Responsibility of Engineers for Alumnus Whistleblowing
}

\author{
Roman Taraban, Edward E. Anderson, M. P. Sharma, and Matthew W. Hayes \\ Texas Tech / Texas Tech / University of Wyoming / Texas Tech
}

\section{Introduction}

College workload - the amount of academic work expected of students-is one of the most important factors affecting students' engagement with their college studies, the quality of instruction and learning, and the drop-out rate. ${ }^{1}$ Workload is determined primarily by college instructors, who control it through the number and nature of assignments in a course-i.e., through the demands they make of students in class and out of class. ${ }^{2}$ Students distinguish good teaching from bad teaching in part by whether they perceive the learning as being deep, engaging, and reflective, versus superficial, passive, and mostly memorization. This latter condition arises when workload is too high. ${ }^{1}$ Therefore, the teacher faces a dilemma. On the one hand, he or she must demand enough of students, ${ }^{2}$ but on the other this will only be effective, and be perceived as effective, if students have sufficient resources to rise to those demands and to fulfill them in a deep fashion. Time is a resource, and an excessive workload can force students to complete tasks superficially. These general instructional issues apply to much of the contemporary development of computer-based and multi-media instructional materials. Computer materials must be developed and tested in ways that assure that they engage students in a deep fashion and that they can be completed within the temporal constraints faced by the students.

The demands of the instructor interact with student characteristics. It is important to know how much time students allocate to study, how they distribute their time among the demands and resources available to them, what motivates students, and which learning resources are of greatest benefit to students. We will show that much can be learned about how to implement materials and measure their effectiveness by monitoring students' study behaviors through questionnaires and dynamic computer records. This kind of information is useful to curriculum developers in the ongoing refinement and improvement of instruction.

\section{A Case Study Involving Texas Tech University and the University of Wyoming}

The research described here is part of an ongoing project to develop computer-based materials and to identify effective teaching and learning methods for engineering students in introductory 
thermodynamics. The data presented here were collected from students at two universitiesTexas Tech and the University of Wyoming - the two universities where the instructional materials and methods are currently being developed. One source of data was a questionnaire (See the Appendix) in which students provided background information and described their study behaviors. The second source of data was from computer records, created over the course of a semester, which automatically recorded students' interactions with on-line problem sets accessed over the World Wide Web (WWW).

The following questions were considered in this case study:

- Did students have sufficient computer resources to use the computer-based materials? It was important to know that the introduction of computer-based materials into the curriculum was not precluded by insufficient computing resources among the students.

- Did students allocate sufficient time to complete the course requirements? College instructors have traditionally expected students to spend two hours studying outside of class for every hour spent in class. In a recent study, students reported mean study times that were closer to one hour of study for every hour in class. ${ }^{3}$ Therefore, it was possible that participants in this cohort would fall short of the university expectation for study time and would report study times closer to three hours per week instead of the expected six hours per week.

- Did students have sufficient time to complete on-line homework assignments? Students were assigned computer-based homework problems (described in detail later) throughout the semester. It was important to establish that the problems were challenging, but not too difficult, and could be completed within the time available to students.

- How important were incentives for getting students to use computer-based materials? Students had access to two sets of electronic materials - the on-line homework problems and a CD supplement to the textbook. One question was whether students would show preference for one over the other. It was possible that they would favor the on-line homework problems because in the current implementation students gained course points for completing those problems, but they did not receive points for using the CD.

- How did students allocate time and resources for test preparation? In a recent study of students' test preparation activities, ${ }^{3}$ students allocated the greatest proportion of time to studying lecture notes $(57 \%)$ and a smaller proportion of time to studying the course textbook (34\%) and working in study groups (11\%). The smallest proportion of time was used at web sites. Students in introductory thermodynamics might allocate their test preparation times differently, giving more attention to the textbook and possibly to information available at computer web sites.

- Were participants' study behaviors complementary or compensatory? The thermodynamics course offered students many learning resources. These could be used in a compensatory fashion-e.g., skip class and read lecture notes on the web site-or in a 
complementary fashion-e.g., go to class, review lecture notes on the web site, complete the on-line homework problems, etc. A correlation analysis using the computer and questionnaire data were used to characterize the behavior of students as complementary or compensatory.

Participants-The participants were 31 students at Texas Tech University and 29 students at the University of Wyoming enrolled in Introduction to Thermodynamics who participated for course credit. One participant from Texas Tech and two participants from the University of Wyoming were excluded from this study because they discontinued submitting on-line homework problems within two weeks of the start of the semester. On-line homework data were analyzed for the remaining 57 participants. Demographic data were available from 26 Texas Tech participants and 23 University of Wyoming participants who completed the questionnaire (See the section, Background Information, in the Appendix). The following descriptive information is based on those 49 students. At Texas Tech, $81 \%(n=21)$ of the participants were male, and at the University of Wyoming, 70\% $(n=16)$ were male. Nearly all participants were Caucasian (Texas Tech: 85\%, $n=22$; University of Wyoming: 91\%, $n=21$ ), with Hispanic ethnicity being the second most frequent (Texas Tech: $8 \%, n=2$; University of Wyoming: 4\%, $n=1$ ). All the participants were engineering majors. All but one participant had achieved at least sophomore status. The mean number of earned college credits for Texas Tech participants was 90.76 (Median $=87.00)$, and for the University of Wyoming participants it was 89.88 (Median = 76.00). The mean number of earned credits in engineering courses for Texas Tech participants was 40.88 (Median $=30.00)$, and for the University of Wyoming participants it was 48.88 (Median $=42.50)$. Texas Tech participants were registered for a mean of 13.54 credit hours (Median $=14.00)$, and University of Wyoming participants for a mean of 16.22 credit hours (Median = 16.00), in the semester when the data were collected. The median expected grade for the course was $B$ at both institutions (on a scale of $F$-lowest to $A$-highest). Based on these demographic data, the participants at both institutions came from similar populations. University of Wyoming students had completed more credit hours in engineering and were carrying a higher workload for the semester.

Materials - The textbook for the course was Thermodynamics: An Engineering Approach, $4^{\text {th }}$ ed., by Cengel and Boles. ${ }^{4}$ Accompanying the textbook was a CD with modules that incorporated active learning exercises, including interactive exercises with immediate feedback, graphical modeling, physical world simulations, and exploration within them. ${ }^{5}$ Computer-based homework problems were written by the second author of this paper for the first ten chapters of Thermodynamics and were made available to students over the World Wide Web. Each chapter was divided into subsections ( range $=2$ to 9), for a total of 52 subsections. Each subsection had either three versions of a problem set (41 of the subsections) or one version of the problem set (11 subsections). Each problem set had exactly five questions. Thus, there were 670 questions in the homework database. The questions were based on material in the textbook, lectures, and the $\mathrm{CD}$ that accompanied the textbook. (To view the problem sets, go to www7.tltc.ttu.edu/thermosite. Enter the system as a TTU student with the username guest and password guest.) A questionnaire was constructed by the first author, and consisted of items about the students' background and how they studied.

Proceedings of the 2002 American Society for Engineering Education Annual Conference \& Exposition Copyright (C2002, American Society for Engineering Education 
Procedure-At Texas Tech, classes met during the fall semester on Tuesdays and Thursdays, and one set of homework problems was typically due for each class. The homework assignments for the entire semester were posted at the course web site at the beginning of the semester, and students could access and complete all the problem sets anytime before the due dates. At the University of Wyoming, classes met on Mondays, Wednesdays, and Fridays. There was more variability in how many problem sets were assigned, when they were assigned, and when they were due, compared to Texas Tech.

Each time a student chose a problem set for a chapter subsection, he or she received a problem set consisting of five questions. If there were multiple versions of the problem set, the system randomly selected one of the sets for the student. The questions contained randomly generated parameters in their statements, so it was unlikely that students received identical problem sets. At Texas Tech, the questionnaire was administered at the web site at the end of the semester; at the University of Wyoming, it was administered on paper at the end of the semester.

\section{Results and Discussion}

Computer Background-In order to assess students' ability to access and use computer technology, several questionnaire items asked about computer background (See the Appendix). Nearly all the participants owned a computer with World Wide Web (WWW) access (Texas Tech: $85 \%, n=22$; University of Wyoming: 78\%, $n=18$ ) and capable of playing CDs (Texas Tech: $92 \%, n=24$; University of Wyoming: $87 \%, n=20$ ). Participants gave a median rating of high for their ability to use computers (Texas Tech: high $=58 \%$, medium $=42 \%$; University of Wyoming: high, 57\%, medium $=39 \%$, low $=4 \%$ ). About half of the participants at both institutions had used CDs for instruction in other courses (Texas Tech: 54\%, $n=14$; University of Wyoming: $44 \%, n=10$ ). Based on these data, the participants at both institutions had similar computer backgrounds. The high level of computer ownership suggested that students had made a commitment to the new technologies, and that they possessed sufficient computer resources and knowledge for computer-based instruction.

Class Attendance and Study Time-At Texas Tech, participants reported spending a mean of 6.62 hours (median $=6.00 \mathrm{~h}$ ) per week studying outside of class, and at the University of Wyoming a mean of 6.00 hours (median $=5.00 \mathrm{~h}$ ). Study time was quite close to the university expectation of six hours, thus these students appeared to follow the "golden rule" of two hours of study outside of class for every hour spent in class. Participants reported high class attendance (Texas Tech: Mean $=82 \%$ of classes attended, Median $=90 \%$; University of Wyoming: Mean = $90 \%$, Median $=90 \%$ ). Therefore, participants were not using study time to compensate for high absences from lectures.

On-Line Homework Performance-The data for the 30 participants at Texas Tech and 27 participants at the University of Wyoming who completed the homework problem sets (but not necessarily the questionnaire) are summarized in Table 1. Students could complete a problem set more than once. Ninety-two percent of the submissions from Texas Tech were unique-i.e. participants submitted the problem set only once; 88\% of the University of Wyoming submissions were unique. Participants at the University of Wyoming completed over three times as many problem sets as Texas Tech students, and Wyoming participants took only about $40 \%$ as long, on 
average, to solve a problem, compared to Texas Tech participants. Overall, the collection and analysis of on-line times allowed us to establish that the problems were not too difficult for students and that the problems could be completed within a reasonable amount of time.

The higher mean scores and the fast solution times in the Wyoming data suggested that solving the problems may have become superficial for students. Because we were striving for deep learning, ${ }^{1}$ this possibility deserved closer examination. Pearson correlation coefficients were calculated using three variables for each participant - total on-line time, the total number of problem sets completed, and the mean score for the problem sets. These correlations were calculated separately for the Texas Tech participants and the Wyoming participants. As shown in Table 2, the correlations between the mean score for the problem sets and the total number of problem sets completed was highly significant for both groups of participants. In other words, in both cases, the more sets a participant completed the higher his or her mean score - the completion of more problem sets resulted in better overall performance. Thus, there is no evidence here to suggest that completion of the problems had become superficial for the University of Wyoming participants. In fact, the additional problem sets appear to have increased their skill level.

Table 1. Mean Performance Measures For On-Line Homework, By Participant

\begin{tabular}{|l|l|l|}
\hline & Texas Tech $(n=30)$ & U Wyoming $(n=27)$ \\
\hline Total Hours For The Semester & 2.60 hours & 3.16 hours \\
\hline Total Number Of Problem Sets Submitted & 15.50 & 46.67 \\
\hline Mean Time Per Problem Set & 10.06 minutes* & 4.06 minutes \\
\hline Mean Time Per Problem & 2.01 minutes & 0.81 minutes \\
\hline Mean Score (Max =10) On Problem Sets & 8.50 & 9.11 \\
\hline
\end{tabular}

*One outlier in the Texas Tech data (7.46 hours for a problem set) and one outlier in the University of Wyoming data (3.02 hours for a problem set) were replaced with the mean time for that problem set, excluding the outliers.

On-Line Homework versus CD-It was possible that participants would essentially ignore the $\mathrm{CD}$ that came with the textbook, even though the $\mathrm{CD}$ had high-quality activities, provided immediate feedback, and included simulations and opportunities to explore pheno mena. This was because course points were not associated with the use of the CD. In contrast, students gained course points for completing the on-line homeworks. Texas Tech students reported a mean of 0.57 hours per week (median $=0 \mathrm{~h}$ ) of CD use. University of Wyoming students reported using the $\mathrm{CD}$ a mean of 0.23 hours per week (median $=0 \mathrm{~h}$ ). The low median times suggested a skewed distribution. Indeed, 58\% of Texas Tech participants and 70\% of University of Wyoming participants reported that they did not use the CD. In contrast, 25 of the 26 (96\%) Texas Tech participants who completed the questionnaire also completed the on-line problem sets. University of Wyoming participants submitted questionnaires anonymously, so we could not determine exactly how many completed on-line problems, however, the percentage was high. Therefore, 
these data support a general claim that availability of instructional materials is a necessary factor but not sufficient in itself to motivate students to use the materials. Students completed the online homework because they received points for doing so. Many students ignored the CD because no incentive was provided to use it.

Table 2. Pearson Correlations ( $r$ ) For On-Line Homework

\begin{tabular}{|l|l|l|}
\hline & Mean Score & Total Sets \\
\hline \multicolumn{2}{|c|}{ Texas Tech Participants $(n=30)$} \\
\hline On-LineTime & .35 & $.54^{* *}$ \\
\hline MeanScore & & $.78^{* *}$ \\
\hline University of Wyoming Participants $(n=27)$ \\
\hline On-LineTime & -.18 & .16 \\
\hline \multicolumn{2}{|c|}{} & $.67^{* *}$ \\
\hline MeanScore & & \\
\hline
\end{tabular}

$* p<.05, * * p<.01$

Distribution of Homework Times-Texas Tech students generally had to complete an on-line problem set for their Tuesday class and one for their Thursday class. University of Wyoming students met on Mondays, Wednesdays, and Fridays, and there was more variability in when online problems were assigned. A graph of on-line times for the University of Wyoming showed no clear pattern-See Figure 1. However, an examination of the distribution of on-line times for Texas Tech students showed a clear pattern of completing problem sets very close to the time they were due (i.e., on Monday through Thursday)-See Figure 2. Even though all the problem sets were available at the beginning of the semester, students chose to complete them when they were due.

Distribution of Study Times for In-Class Tests-Participants' responses to questions about their test preparation behaviors (See the Appendix) provided information about how students allocated their time to the several resources that were available to them. Table 3 shows that University of Wyoming participants allocated a greater portion of their total study time for test preparation. The use of study time is quite similar for Texas Tech and University of Wyoming participants. The largest proportion of time was devoted to studying the textbook, followed by studying lecture notes and working in study groups. Only a small proportion of study involved activity at computer web sites. (Note, that the activities are not mutually exclusive-e.g., a person could use lecture notes in the context of a study group-therefore the sum of the percentages for test preparation activities may exceed 100\%). The mean percentages in Table 3 were converted to time in Table 4, based on a mean total study time per week of 6.62 hours for Texas Tech participants, and a mean of 6.00 hours for University of Wyoming participants. In general, the most valuable test preparation resources for these students, as reflected in the 
Figure 1. Total On-Line Times By Day For University of Wyoming Participants

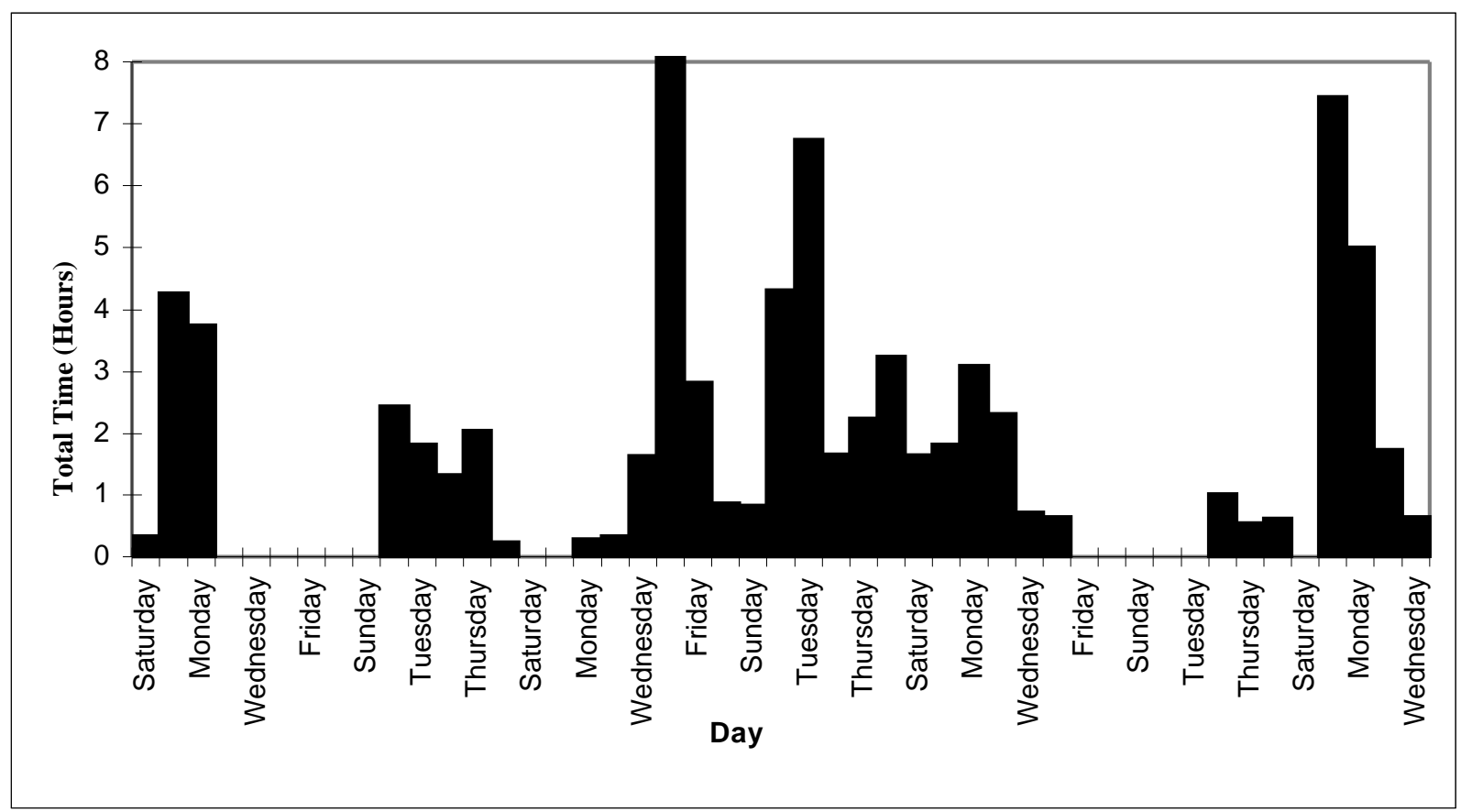

Figure 2. Total On-Line Times By Day For Texas Tech Participants.

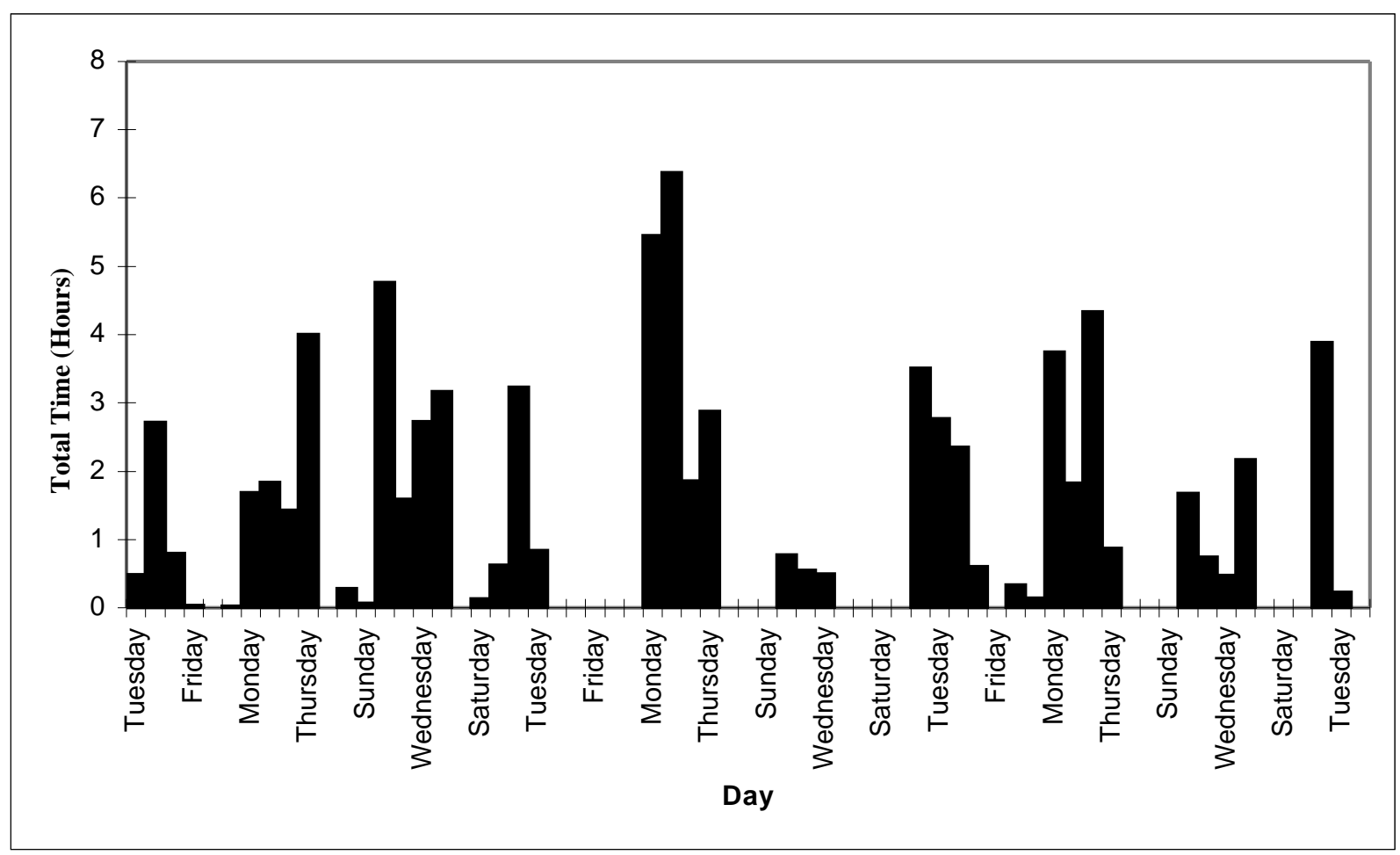


Table 3. Mean (Median) Percent Distributions of Test Preparation Activities

\begin{tabular}{|l|l|l|}
\hline & Texas Tech $(n=26)$ & U Wyoming $(n=23)$ \\
\hline Percent of Total Study Time For Test Preparation & $33.58(25.00)$ & $46.96(50.00)$ \\
\hline Percent of Test Study Time For Lecture Notes & $30.46(22.50)$ & $25.00(20.00)$ \\
\hline Percent of Test Study Time For Textbook & $44.65(40.00)$ & $48.48(50.00)$ \\
\hline Percent of Test Study Time At Websites & $6.19(1.00)$ & $7.83(5.00)$ \\
\hline Percent of Test Study Time For Study Group & $19.69(3.50)$ & $29.13(20.00)$ \\
\hline
\end{tabular}

Table 4. Mean Hours Per Week For Test Preparation Activities

\begin{tabular}{|l|l|l|}
\hline & $\begin{array}{l}\text { Texas Tech }(n= \\
26)\end{array}$ & U Wyoming $(n=23)$ \\
\hline Study Time For Test Preparation & 2.22 & 2.82 \\
\hline Test Study Time For Lecture Notes & 0.68 & 0.71 \\
\hline Test Study Time For Textbook & 0.99 & 1.37 \\
\hline Test Study Time At Websites & 0.14 & 0.22 \\
\hline Test Study Time In Study Groups & 0.44 & 0.82 \\
\hline
\end{tabular}

proportion of time allocated, were the textbook, followed by lecture notes and study groups. Information at websites played a very minor role.

Complementary Versus Compensatory Study Behaviors-In order to test whether participants used course resources in a complementary or compensatory manner, we selected three variables that could reveal one or the other type of behavior - total on-line time for homework problem sets, self-reported class attendance rates, and self-reported weekly study times (See the Appendix for questions for the second and third variables). Because the questionnaire data were collected anonymously from University of Wyoming participants, these variables could not be correlated for that group. Pearson correlation coefficients are displayed in Table 5. The pattern of positive correlations distinguished between the "good" students who attended class, did the on-line homeworks, and studied, and the "weak" students who did less of all these behaviors. Compensatory behaviors would have been evident in negative correlations, which were not present-e.g., between attendance and study time, indicating that students who had low attendance compensated by studying more, and vice versa. 
Table 5. Pearson Correlations ( $r)$ For Texas Tech Participants $(n=26)$

\begin{tabular}{|l|l|l|}
\hline & Attendance & Total Study Time \\
\hline On-LineTime & .31 & $.43^{*}$ \\
\hline Attendance & & $.52 * *$ \\
\hline
\end{tabular}

\section{Conclusions}

College instructors face a dilemma in designing instruction. On the one hand, they must demand enough of students ${ }^{2}$, but on the other this will only be effective, and be perceived as effective, if students have sufficient resources to rise to those demands and to fulfill them in a deep fashioni.e., one that goes beyond passive learning and rote memorization. ${ }^{1}$ In this case study, we showed how questionnaire data and dynamic computer records that document students' interactions with course materials could be used to determine whether students are allocating sufficient material and temporal resources to complete academic tasks in a deep fashion.

Students' reports that they did not use the CD suggested that they needed course-related incentives to use these computer-based materials. It is worth noting that the Cengel and Boles textbook ${ }^{4}$ used in this study is well-organized and may be particularly well-suited to successful completion of the kinds of short on-line homework problems that were assigned to students in this study. If the text were organized differently, or if students found the material particularly difficult for other reasons, they may have used the $\mathrm{CD}$ more for additional learning assistance.

The data also suggested that students used learning resources in a complementary manner, with one activity, like attending class, supporting other activities, like studying. There was no evidence that students were trading off resources in a compensatory manner, like studying more because of high class absenteeism. We will continue to use these methods to monitor student behaviors as we refine the existing materials and expand the computer resources for this thermodynamics course.

\section{References}

[1] Chambers, E. Studies in Higher Education, 1992, 17, 141-153.

[2] Thomas, J., Bol, L., Warkentin, R., Wilson, M., Strage, A., \& Rohwer Jr., W. Applied Cognitive Psychology, 1993, 7, 499-532.

[3] Taraban, R., \& Hayes, M. Measuring Study Time in College. Manuscript submitted for review.

[4] Cengel, Y. A. and Boles, M. A. Thermodynamics: An Engineering Approach, $4^{\text {th }}$ ed.; McGraw-Hill: Boston, MA, 2001.

[5] Anderson, E. E., Sharma, M. P., Taraban, R. Application of Active Learning Technique s. Manuscript submitted to the 2002 American Society for Engineering Education Annual Conference \& Exposition. 


\section{Appendix-Questionnaire Used in the Case Study}

The textbook for the course, Thermodynamics, by Cengel and Boles, comes with a CD that contains additional thermodynamics study materials. Your responses to this questionnaire will be used to assess and to improve the CD materials. Your responses will not be used in any way to determine your course grade. Please be as honest as possible in responding. Your responses will be held in strict confidence. You will not be personally associated with your responses in any discussion or presentation of students' responses to these questions.

\section{Background Information}

1. Gender a) Male b) Female

2. Ethnicity a) Caucasian b) Hispanic c) African-American d) Asian e) American Indian f) Other

3. Are you an engineering major? a) yes b) no

4. Total number of earned college credit hours

5. Total number of earned credit hours in engineering

6. Total number of hours enrolled this semester

7. Your expected grade in this course is a) A b) B c) C d) D e) F

\section{Computer Background}

8. Do you own a personal computer with WWW access? a) yes b) no

9. Do you own a personal computer capable of playing CDs? a) yes b) no

10. How would you rate your ability to use computers? a) low b) medium c) high

11. Have you used a CD for instruction in other courses? a) yes b) no

\section{About This Course}

12. On average, what was your attendance rate for this class (100\% means perfect at tendance)? $\%$

13. On average, how much time did you spend each week to study or work on assignments for this class? Do not include time spent in class, an organized lab, or discussion section. hours

14. Think about how much time you reported studying in the previous question (\#13).

A. What percent of you total Study time did you use to study for tests? (Choose any number between 0\% and $100 \%$.) $\%$

B. When you prepared for a test, what percent of your total time did you spend studying your lecture notes? (Choose any number between $0 \%$ and $100 \%$.) $\%$

C. When you prepared for a test, what percent of your total time did you spend studying your textbook? (Choose any number between $0 \%$ and $100 \%$.) $\%$ 
D. When you prepared for a test, what percent of your total time did you spend at a computer web site? (Choose any number between $0 \%$ and $100 \%$.) $\%$

E. When you prepared for a test, what percent of your total time did you spend with a study group (one or more classmates)? (Choose any number between $0 \%$ and $100 \%$.) $\%$

F. What were your other sources of information when preparing for a test? (List them here.)

15. My opinion of the course content is a) very low b) low c) neutral d) high e) very high

16. My feelings about the course in general are a) very negative b) negative c) neutral d) positive e) very positive

\begin{abstract}
About the CD
17. A CD was included with your textbook. How useful do you think the CD was to learn the course material and do well on the tests? a) not at all useful b) somewhat useful c) useful d) very useful e) exceptionally useful

18. A CD was included with your textbook. On average, how much time did you spend each week using the CD? hours
\end{abstract}

\title{
ROMAN TARABAN
}

Roman Taraban is Associate Professor in the Department of Psychology at Texas Tech University. He received his $\mathrm{Ph} . \mathrm{D}$. in cognitive psychology from Carnegie Mellon University. His interests are in how undergraduate students learn, and especially, how they draw meaningful connections in traditional college content materials (e.g., textbooks, lectures, multi-media).

\section{EDWARD E. ANDERSON}

Edward E. Anderson is Professor of Mechanical Engineering at Texas Tech University where he is also the Associate Director of the University Teaching, Learning, and Technology Center. His responsibilities at the Center are to train and assist faculty throughout the university in applying technology to their teaching. He received his B.S. and M.S. degrees in Mechanical Engineering from Iowa State University and Ph.D. degree from Purdue University.

\section{P. SHARMA}

M. P. Sharma is Professor of Chemical and Petroleum Engineering at University of Wyoming. He received his $\mathrm{Ph} . \mathrm{D}$. degree in Mechanical Engineering from Washington State University. A current area of interest is conducting research on teaching and learning methods, especially on the use of synchronous and asynchronous tools using Web technology.

\section{MATTHEW W. HAYES}

Matthew Hayes is a graduate student in the Experimental Psychology program at Texas Tech University.

This research was supported, in part, by a grant from the National Science Foundation, NSF-CCLI 0088947. We are grateful to Monica Wall for assistance with programming the computer materials and database, and for comments on this paper provided by an anonymous reviewer. 